\title{
Modelo de demanda para simuladores interactivos de cadenas de suministro.
}

\author{
Lourdes Perea Muñoz ${ }^{1, *}$, Víctor Herrero ${ }^{1}$, Alejandro Clausse ${ }^{2}$. \\ ${ }^{1}$ Universidad Austral-CONICET, Buenos Aires, Argentina \\ (lperea, vherreo) daustral.edu.ar \\ ${ }^{2}$ CNEA-CONICET y Universidad Nacional del Centro, Tandil, Argentina
}

\begin{abstract}
Resumen. Se ha desarrollado un modelo de simulación de demanda en el mercado con preferencia de proveedor según la performance de servicio. En las últimas décadas, luego de la aparición del juego de la cerveza en el MIT, se construyeron muchos simuladores de cadenas de suministro con fines educativos y de investigación. Sin embargo pocos de ellos proponen un modelo de competencia entre cadenas, centrándose sólo en la performance de la misma. El resultado presentado es una mejora aplicable a los juegos ya existentes, en particular para DLS (DistributedLogisticSimulator). El modelo sigue el comportamiento del mercado tanto en la preferencia de proveedores como en la cantidad solicitada, habiendo definido el nivel de servicio proporcional a la inversa del tiempo de satisfacción de los pedidos y asumiendo que todos los pedidos se satisfacen en algún momento. El modelo tiene especialmente en cuenta el tiempo máximo que un cliente está dispuesto esperar, no importa lo barato que cueste el producto y el precio máximo que pagaría aunque la entrega sea inmediata.
\end{abstract}

Palabras clave: Modelo de demanda, cadenas de suministro, simulación

\section{$1 \quad$ Introducción}

La tendencia en el mercado actual es que los negocios no compiten en forma aislada sino que están integrados en cadenas complejas. La gestión de las relaciones a través de los componentes de estas cadenas es a lo que se llama, de manera ya extendida, "Gestión de la Cadena de Suministro" (GCS). Lambert [1] lo define como la "integración de los procesos clave de negocio desde el usuario final hasta los proveedores iniciales, que provee productos, servicios e información, que agrega valor a los clientes y a otras personas influidas. El entendimiento de esta interacción dinámica y de los mecanismos de integración son elementos clave en la creación de valor.

Esta realidad del mundo de los negocios, supone un importante desafío para las universidades, ya que la naturaleza dinámica de las cadenas hace que los métodos tradicionales de enseñanza no sean suficientes a la hora de transmitir los conceptos de la GCS. Según Sterman [2] sería equivalente a exponer a un piloto a volar un jumbo jet sin haber pasado antes por largos períodos de entrenamiento en un simulador. Así, con la incorporación de las TICs, los simuladores surgen como complemento para 
cursos de Gestión Logística y Cadena de Suministro (CS) aportando entre otros los siguientes beneficios:

- Ayudan a conectar la teoría y la práctica para fomentar la comprensión de los alumnos.[3]

- Abren la participación dinámica, lo que debería reducir la resistencia a aceptar ideas y conceptos innovadores.[4]

- Dan retroalimentación inmediata al estudiante, haciendo que la interacción con el juego sea un proceso de aprendizaje más que un proceso de evaluación. [5]

- Dan al estudiante la posibilidad de hacer frente a las consecuencias de los resultados de las decisiones tomadas y no ser solo un mero observador.[6]

- Estas herramientas de aprendizaje a través de la experiencia multi-sensorial permite al jugador experimentar la cooperación y el trabajo en equipo sin el riesgo de incurrir en errores a veces costosos.[7]

Los juegos de simulación suelen tener un objetivo muy concreto de enseñanza, lo que lleva a realizar simplificaciones de la realidad en orden a focalizarse en ese punto. "No existe ningún juego que pueda aplicarse con éxito a todas las situaciones de enseñanza. Por el contrario, la situación practica y las necesidades de educación varían tanto que hacen necesarios diferentes juegos" [8]. Sin embargo, hay que tener cuidado para que estas simplificaciones no introduzcan distorsiones de la realidad que puedan llevar a los alumnos a conclusiones desacertadas. "Cuanto más realista sea "el mundo del juego", más efectiva será la simulación.” [9]

En la sección 2 nos centraremos en la descripción de los juegos de simulación desarrollados que consideramos más relevantes, por sus contribuciones y el interés despertado por otros autores, sin embargo nos interesa subrayar de manera adelantada que la mayoría de ellos no plantea una competencia real entre cadenas por el mercado. El mercado en estos casos está dado por una demanda (con diferentes características de acuerdo al juego) no influenciada por el comportamiento de la cadena y de su competencia. Así centran su objetivo en maximizar ganancias o minimizar costos logísticos olvidando que toda cadena es en última instancia manejada por el mercado.

Zhang [10] advierte que ciertos problemas de competencia internos de la cadena son investigados a través de modelos teóricos de juegos, pero hay muy pocos casos que estudien la interacción de múltiples cadenas y en particular la competencia de Cadena de Suministro versus Cadena de Suministro "CS vs CS", a pesar de un reconocimiento generalizado de que la competencia actual no es ya empresa versus empresa sino entre cadenas. Plantea también las siguientes preguntas: ¿De qué manera compiten las CS?; ¿Qué CS ganará la competencia?; ¿Que porción del mercado ("Market Share") podrá obtener una CS ganadora?

Por otro lado, hemos encontrado, algunos modelos de demanda aplicados a juegos de simulación de negocios [11]. El planteo en estos es la asignación de recursos al "Marketing Mix" de modo de lograr la mayor cuota de mercado, pero sin tener en cuenta los costos logísticos asociados a la puesta en el mercado del producto. Encontramos en estos modelos ideas interesantes para plantear nuestro modelo de competencia entre cadenas de suministro. 
En la sección 3 describimos el DLS (Distributed Logistic Simulator), simulador para el cual desarrollamos el modelo de demanda que se presenta en este trabajo. DLS fue desarrollado con fines de entrenenamiento de operadores de cadena, aunque presenta un alto potencial para optimizar decisiones en una cadena, la que deberá ser validada con datos reales.

\section{Juegos de simulación de la CS y Modelos de Demanda}

Una rápida selección entre los simuladores existentes nos permitió quedarnos con los más conocidos y representativos. En la Tabla 1 se muestra una síntesis del objetivo de cada juego y una breve descripción de su alcance y tipo de desiciones representadas.

Table 1. Lista de los juegos de Logística y Cadena de Suministro

\begin{tabular}{|c|c|c|c|}
\hline Nombre del Juego & Descripción & Decisiones/Alcance & Desarrollado por \\
\hline $\begin{array}{l}\text { MIT Beer Game } \\
\text { (www.beergame.mit.edu) }\end{array}$ & $\begin{array}{l}\text { Cadena de suministro de } \\
\text { cerveza de } 4 \text { eslabones } \\
\text { que atiende los pedidos } \\
\text { del mercado }\end{array}$ & $\begin{array}{l}\text { Asumir los roles de: Fábrica, Mayorista, Distribuidor o } \\
\text { Minorista y determinar las cantidades a pedir. Objetivo: } \\
\text { minimizar el costo logístico total, teniendo en cuenta } \\
\text { costo de inventario y penalización por ordenes } \\
\text { pendientes }\end{array}$ & $\begin{array}{l}\text { Massachusetts } \\
\text { Institute of technology, } \\
\text { USA (1988) }\end{array}$ \\
\hline Columbia Beer Game & $\begin{array}{l}\text { Producción y Distribución } \\
\text { de Cerveza en una cadena } \\
\text { de múltiples eslabones }\end{array}$ & $\begin{array}{l}\text { Asumir los roles de: Fábrica, Mayorista, Distribuidor o } \\
\text { Minorista. Objetivo: Similar al Beer Game pero con } \\
\text { demanda estocástica }\end{array}$ & $\begin{array}{l}\text { Columbia University } \\
(1999)\end{array}$ \\
\hline $\begin{array}{l}\text { HuliaGame } \\
\text { (www.hulia.haifa.ac.il/Eng/ } \\
\text { hulia.html) }\end{array}$ & $\begin{array}{l}\text { Producción y Distribución } \\
\text { de Cerveza en una cadena } \\
\text { de múltiples eslabones }\end{array}$ & $\begin{array}{l}\text { Determinar las estrategias en cadenas de multiples } \\
\text { niveles. Actuar como Minoristas, Mayoristas, etc para } \\
\text { determinar las cantidades de pedido. Objetivo: idem } \\
\text { Beer Game }\end{array}$ & $\begin{array}{l}\text { The University of } \\
\text { Haifa, Israel. (2000) }\end{array}$ \\
\hline $\begin{array}{l}\text { Trading Agent Competition } \\
\text { http://www.sics.se/tac }\end{array}$ & $\begin{array}{l}\text { Agentes de Comercio que } \\
\text { responden a las } \\
\text { licitaciones de PCs de los } \\
\text { clientes }\end{array}$ & $\begin{array}{l}\text { Ganar las licitaciones de los clientes y ser capaces de } \\
\text { coordinar eficazmente el aprovisionamiento, compras, } \\
\text { producción, y decisiones sobre plazos y precios. } \\
\text { Objetivo: obtener la máxima posición de ganancia. }\end{array}$ & $\begin{array}{l}\text { Swedish Institute of } \\
\text { Computer Science } \\
\text { (2003) }\end{array}$ \\
\hline $\begin{array}{l}\text { The Supply Chain Game } \\
\text { http://www.responsive.net }\end{array}$ & $\begin{array}{l}\text { Red de Suministro de un } \\
\text { producto químico }\end{array}$ & $\begin{array}{l}\text { Controlar una empresa virtual, que provee un producto a } \\
\text { fabricantes de aires acondicionados, gestionando y } \\
\text { ampliando la red de suministros a través de un } \\
\text { continente ficticio. Objetivo: Obtener la máxima posición } \\
\text { de ganancia }\end{array}$ & \begin{tabular}{|l|} 
Kellogg School of \\
Management at \\
Northwestern \\
University. (2005)
\end{tabular} \\
\hline $\begin{array}{l}\text { The Logi-Game } \\
\text { http://www.moltho.dk }\end{array}$ & $\begin{array}{l}\text { Gestion de materiales a } \\
\text { través de un Canal de } \\
\text { Distribución de bicicletas }\end{array}$ & $\begin{array}{l}\text { Actuar como fabricantes, mayorista o minorista tomando } \\
\text { decisiones de manufactura y de inventario. Objetivo: } \\
\text { lograr el mayor nivel de servicio al menor costo posible. }\end{array}$ & $\begin{array}{l}\text { Technical University of } \\
\text { Denmark (2009) }\end{array}$ \\
\hline $\begin{array}{l}\text { SIMPLE } \\
\text { http://scmlab.nctu.edu.tw:8 } \\
\text { 084/Esimplex/ }\end{array}$ & $\begin{array}{l}\text { Producción y Distribución, } \\
\text { dentro de una cadena } \\
\text { permitiendo la } \\
\text { configuración flexible de } \\
\text { la misma }\end{array}$ & $\begin{array}{l}\text { Asumir los diferentes roles dentro de la cadena llevando } \\
\text { a cabo la gestión de Inventario, gestion de capacidad, } \\
\text { determinación de precio y negociación. Permite } \\
\text { compartir información entre los distintos eslabones. } \\
\text { Objetivo: lograr el mayor nivel de servicio al menor costo } \\
\text { posible. }\end{array}$ & $\begin{array}{l}\text { National Chiao Tung } \\
\text { University (2009) }\end{array}$ \\
\hline
\end{tabular}

De todos los juegos, el más extendido y conocido es el Juego de la Cerveza [12], cuyo objetivo es mostrar las ventajas de una visión integral en la GCS. En particular, el valor de compartir información entre los componentes que integran la cadena. Luego de la aparición del juego de la cerveza, diversos y numerosos juegos fueron apareciendo y fueron adoptados no sólo en las aulas para facilitar el aprendizaje sino también en otras circunstancias para estimular la investigación o para introducir la competencia internacional y la colaboración entre estudiantes, profesores e investigadores. Así, por ejemplo, el Columbia Beer Game, se utilizó para modelar una cadena computarizada manejada por agentes artificiales e investigar si estos agentes pueden lograr 
mejores resultados que los humanos jugando al Juego de la Cerveza [13]. Y el caso de Trading Agent Competition con el que se organizan competencias internacionales con fines de investigación.

Respecto a la simulación del Mercado, en la mayoría de los casos se toma una situación de demanda como dada e igual para todas las cadenas. La falta de disponibilidad es percibida como fundamental en la performance de la cadena y por ende se penaliza con mayor énfasis, por ejemplo, que el inventario mantenido. Sin embargo la demanda de la cadena no se ve afectada a futuro por la falta de entrega, como tampoco por las estrategias o el buen desempeño de la competencia. Es decir, el problema se centra en la minimización de los costos logísticos dada una demanda inalterada por las decisiones de la cadena.

Si bien son diferentes en cuanto a la diversidad de decisiones y complejidades de los sistemas, podemos citar en esta línea al Beer Game del MIT, el Hulia Game [9], el Columbia Beer Game, The Logi Game [14, 15] y SIMPLE [16].

The Supply Chain Game [17] y Trading Agent Competition [18], presentan un enfoque diferente. En el caso del primero el problema es de red de distribución, con pérdida de pedidos por incumplimiento en la entrega, aunque esto no afectará la demanda futura. En el caso de "Trading Agent Competition", se plantean problemas de competencia entre cadenas tanto aguas arriba, por el aprovisionamiento de componentes, como hacia abajo, por las licitaciones de los clientes finales. Sin embargo, en lo que respecta a demanda, a pesar de plantear la competencia por un pedido en particular, la performance histórica del agente no es tenida en cuenta por el cliente a la hora de asignar un nuevo pedido.

\section{$3 \quad$ DLS (Distributed Logistic Simulator)}

DLS (Distributed Logistic Simulator), fue desarrollado en la UNCPBA, bajo la dirección de los Dres. Herrero y Clausse. Diseñado para promover el entrenamiento y desarrollo de operadores de CS. Los jugadores pueden aplicar y testear diferentes estrategias en orden a adquirir el mayor nivel de servicio al menor costo posible.

Si bien, el Beer Game resulta muy útil para resaltar los efectos de fallas sistémica y amplificación de los errores por retroalimentación. El DLS, agrega: mayor detalle y realismo en las variables de decisión del entrenando; flexibilidad en la construcción de las cadenas; y posibilidad de competencia real por la participación en el mercado, a través del Modelo de Demanda que introduciremos en la sección 4.

El sistema permite configurar diferentes tipos de cadenas de manera muy flexible, mediante la composición de 4 elementos o componentes básicos:

- Almacenes (A) y Operadores de Zona de Picking (OZP): Constituyen las estaciones interactivas simulando el escenario logístico. Hacen las veces de Mayoristas, Minoristas, Distribuidores, etc. Son manejadas por los entrenandos.

- Fábricas (F) y Mercado: Simulan el mundo exterior. El Administrador o Instructor establece las condiciones contorno de manera externa y dinámica. 


\subsection{Descripción de los diferentes componentes:}

Componente Fábrica: interactúa con los almacenes de los primeros proveedores de la cadena simulando, estadísticamente, demoras en las entregas e imponiendo el precio de fábrica.

Componente Cliente: Interactúa con los operadores de zona de picking correspondientes a los diferentes retailers (último eslabón) de las distintas cadenas. Genera aleatoriamente pedidos de productos y simula preferencias por precio y performance de entrega, de acuerdo al "Modelo de Demanda" establecido.

Componente Almacén: de acuerdo al rol desempeñado dentro de la cadena, interactúa con OZP aguas abajo, para el armado de los pedidos solicitados por los clientes; o recibe productos para almacenar de sus proveedores. Entre otras cosas el entrenando deberá definir la estrategia de almacenamiento y asignación a las diferentes zonas, teniendo en cuenta los costos asociados. A su vez, deberá establecer la estrategia de reposición y el valor de los parámetros de planificación (tamaño de lote, SS, etc.) El entrenando cuenta con un panel de control en el que puede monitorear las diferentes variables del sistema (stock, costos, tiempos de servicio, productos en espera, etc.)

Componente OZP: deberá atender a los pedidos de sus clientes (cliente final o almacén aguas abajo) a través de diferentes estrategias, cómo la asignación de prioridades de entrega de las ordenes (FIFO, FDFO) y controlando también la cantidad de mano de obra (con sus costos asociados) y el tipo de asignación de pedidos a los operarios. En su panel de control el operario monitorea las diferentes variables (pedidos, nivel de ocupación de operarios, performance operativa y económica, etc.)

\subsection{Configuración de escenarios:}

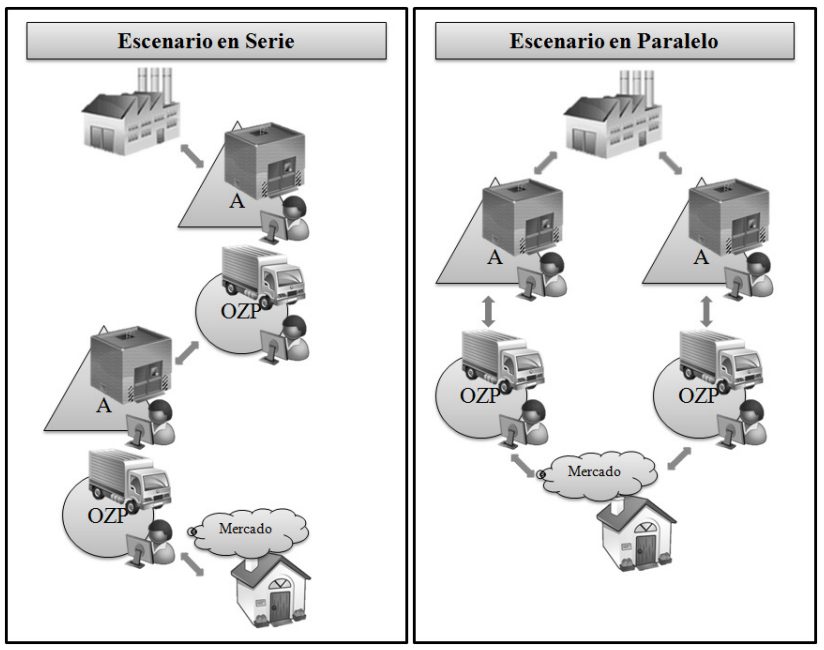

Fig. 1.Escenario en serie y en paralelo. 
Mediante la combinación de estos 4 componentes, se pueden establecer diferentes escenarios. Hay dos que interesan en particular a los fines de enseñanza del juego:

Escenario en Serie: permite al entrenando testear las diferentes estrategias aplicables y ver cómo impactan en una situación de mercado determinada. Sería el caso similar al planteado por el beer game, Logi Game, SIMPLE.

Escenario en Paralelo: en este escenario dos cadenas compiten por el mismo mercado. Aquí no sólo deberán tener en cuenta sus propias estrategias sino también las de sus competidores y las fluctuaciones en la demanda. Permite ver cómo opera el Modelo de Preferencia. La Figura 1 muestra ambos escenarios, en el primer caso un escenario en serie, con dos eslabones de A- PZO (uno mayorista y otro minorista) y en el segundo la competencia de dos minoristas.

\section{$4 \quad$ Modelo de demanda propuesto}

\subsection{Modelo de Comportamiento del Mercado:}

El modelo desarrollado queda definido por las siguientes 4 ecuaciones:

$$
\begin{aligned}
& \text { 1. Precio del mercado como la Media Armónica del precio de todos los } \\
& \text { proveedores. } \\
& \bar{P}=\# r e t / \sum_{i=1}^{\# r s t}\left(1 \mid \bar{p}_{i}\right) \\
& \mu_{n}=\mu_{n-1}-k \Delta \overline{\mathrm{P}} \\
& w_{i}=\left\{\begin{array}{r}
\text { Pref }_{i}=\left(1-\frac{P_{i}}{P_{\max }}\right) *\left(1-\frac{\bar{\tau}_{i}}{\tau_{\max }}\right) \\
\text { siPref }_{\mathrm{i}}>0 \\
0 ; \text { siPref } f_{\mathrm{i}} \leq 0
\end{array}\right. \\
& M S i=\frac{w_{i}}{\sum_{i=1}^{* \beta \varepsilon t} w_{i}}
\end{aligned}
$$

\subsection{Desarrollo del Modelo}

Media armónica para el precio promedio:

Para obtener el precio promedio del mercado $(\bar{P})$, utilizamos la media armónica, coincidiendo con Gold \&Pray [11], en que ésta asigna un peso relativo mayor a los precios más bajos que a los altos, y por tanto es más coherente con la teoría económica, ya que, las empresas con precios más bajos generarán mayores cantidades demandadas que aquellas de mayor precio. La fórmula utilizada es:

$$
\bar{P}=\# \text { ret } / \sum_{i=1}^{\# r e t}\left(1 \mid \mathrm{P}_{i}\right)(1)
$$

Donde, \#ret, es la cantidad de retailers totales y $\mathrm{P}_{\mathrm{i}}$ es el precio del retailer "i". 


\section{Media del mercado corregida por variación de precios:}

El mercado será simulado de acuerdo a una distribución de probabilidad cuyos parámetros estadísticos $(\mu ; \sigma)$, son fijados inicialmente por el instructor, respondiendo a la forma que más se adapte al mercado simulado (en general, Poisson $\left(\mu_{0}\right)$ y Normal $\left(\mu_{0} ; \sigma\right)$. Sin embargo, esta demanda media se verá afectada al variar los precios de los retailers. Si el precio promedio del mercado se incrementa, la cantidad demandada sufrirá un decremento y viceversa. Por tanto:

$$
\mu_{n}=\mu_{n-1}+\Delta \mu
$$

donde $\mathrm{n}$ es el paso temporal actual y n- 1 el paso temporal anterior.

Si suponemos una función de demanda en función del precio rectilínea, es decir:

$$
\mu=\mu_{\max }-k \bar{P}
$$

con $\mu_{\max }$ igual al valor máximo que puede tomar la demanda en $\mathrm{P}$ tendiendo a 0 . Luego k, estará dado por:

$$
k=\left(\mu_{\max }-\mu_{0}\right) / P_{0}
$$

El valor máximo de demanda $\left(\mu_{\max }\right)$ en $\mathrm{P}$ tendiendo a cero, así como las condiciones iniciales e iguales para todos los retailers (Po y $\left.\mu_{0}\right)$ son fijadas por el instructor.

Así, en cada paso temporal, la media será actualizada según:

$$
\mu_{n}=\mu_{n-1}-k \Delta \overline{\mathrm{P}} ;\left(\operatorname{con} \Delta \bar{P}=\bar{P}_{n}-\bar{P}_{n-1}\right)(7)
$$

La demanda efectiva $\mathrm{Q}_{\mathrm{n}}$ de ese paso temporal será, por tanto:

$$
\mathrm{Q}_{n}=\mathrm{f}\left(\mu_{n} ; \sigma\right)
$$

Pesos de los diferentes retailers en función de precio y nivel de servicio histórico: Coincidimos con Tsay y Agrawal [18] en que la percepción de valor del cliente y por ende su decisión de compra no estará dada sólo por el precio del producto sino también, por la cantidad de servicio que lo acompaña, de modo que un retailer que reduzca su precio o aumente su servicio gozará de un incremento en sus ventas. Definimos el nivel de servicio como la inversa del tiempo de entrega: $\mathrm{NSi}=1 / \tau \mathrm{i}$

De este modo, el modelo de preferencia asigna las órdenes a las diferentes cadenas basado en el precio y el nivel de servicio histórico de cada una. De acuerdo a la ecuación de preferencia del cliente y pesos relativos de los diferentes retailers.

$$
w i \quad\left\{\begin{array}{c}
\operatorname{Pref}_{i}=\left(1-\frac{P_{i}}{P_{\max }}\right) *\left(1-\frac{\bar{\tau}_{i}}{\tau_{\max }}\right) ; \text { si Pref }_{i}>0 \\
0 \quad ; \operatorname{siPref}_{i} \leq 0
\end{array}\right.
$$

El peso relativo de cada retailer y por ende su probabilidad de recibir órdenes estará dada por el precio ofrecido por el mismo $\left(\mathrm{P}_{\mathrm{i}}\right)$ para el pedido en particular y por su desempeño de entrega histórico $\left(\bar{\tau}_{i}\right)$. Teniendo en cuenta el tiempo máximo $\left(\tau_{\max }\right)$ que un cliente está dispuesto esperar, no importa lo barato que cueste el producto y el precio máximo $\left(\mathrm{P}_{\max }\right)$ que pagaría aunque la entrega sea inmediata.

\section{Market Share (Ms).}

Por último la cantidad demandada a cada retailer en un paso temporal será:

$$
Q_{i=} Q . M S i \text { con } \mathrm{Q}=\mathrm{f}\left(\mu_{n} ;\right. \text { parámetros estadísticos) (10) }
$$


Donde el Market Share del retailer i (MSi) será su peso relativo normalizado:

$$
M S i=\frac{w_{i}}{\sum_{i=1}^{\# P Z O} w_{i}}(4)
$$

\section{Comportamiento del modelo:}

\subsection{Respuesta a diferentes cambios en Precio y Nivel de Servicio.}

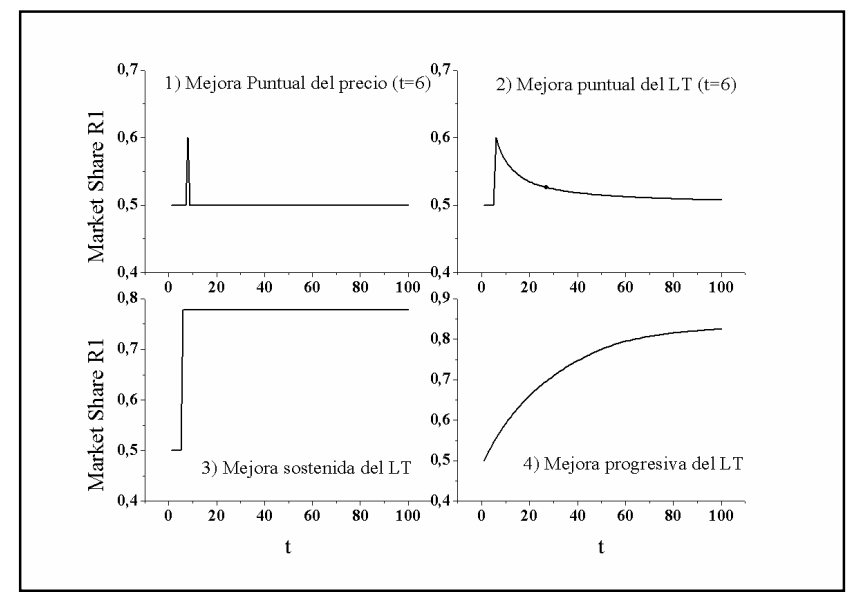

Fig. 2. Evolución del MS de un retailer ante cambios en precio y LT.

Analizamos diferentes situaciones para mostrar el comportamiento del sistema. La figura 2 muestra la respuesta del sistema ante diferentes cambios en el Precio y el tiempo de entrega LT $(\tau)$ de un retailer $(\mathrm{R} 1)$ en competencia con otro. Ambos parten con las mismas condiciones iniciales, manteniéndolas uno de ellos (R2) durante toda la simulación. Podemos ver como el MS del R1 se incrementa, tanto al reducir su precio en un paso temporal (1), como al reducir su LT (2), aunque en el primer caso sólo se beneficiará un instante mientras que en el segundo mantendrá cierto beneficio en el tiempo, hasta que pasado un lapso considerable recuperará la posición de mercado inicial. Esto responde al hecho de que el cliente considera el precio para la compra en particular, mientras que tiende a tener una mayor confianza en aquel retailer que le brinda un mejor servicio. Para mantener la posición ganada por una mejora del LT, el retailer deberá ser persistente en esta mejora en el tiempo (3). Por último, se ve cómo se va incrementando el MS (o ventas) de un retailer a medida que mejora su NS respecto de sus competidores (4), hasta llegado un punto en que el cliente ya no saque beneficio de esta mejora y por ende su nivel de ventas permanecerá constante. 


\subsection{Comportamiento del modelo: Media armónica de precios vs. Media aritmética}

La media armónica asigna un mayor peso relativo a los precios más bajos. En la Fig. 3 se puede apreciar la variación que producen en la media armónica los precios bajos de uno u otro retailer, para una media aritmética constante. Y cómo el sistema responde asignando un mayor MS a aquel retailer que ofrece realmente el precio más bajo. Para el caso expuesto, una media aritmética hubiera hecho permanecer el precio constante de modo que no se produciría ninguna variación en la cantidad demandada a pesar de que algún retailer ofreciera un precio realmente atractivo.

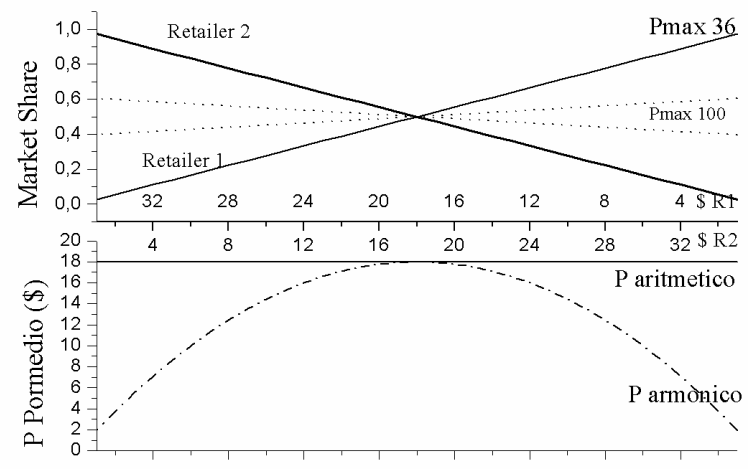

Fig. 3. Variación del MS de los retailers y la media armónica en función de la variación de precios, para un promedio aritmético constante.

\section{Conclusiones}

El Comportamiento del Modelo de Demanda para juegos de Simulación de Cadenas de Suministro, demuestra que tiene la capacidad de modelar la generación de la Demanda Total del mercado, teniendo en cuenta la ley de demanda, cómo también la de simular la Demanda Particular de cada cadena de acuerdo a las preferencias de los clientes. Esta última, basada en que la percepción de valor por parte de los clientes no estará dada sólo por el precio, sino que también es influenciada por el nivel de servicio que lo acompaña. El tener en cuenta el tiempo máximo que un cliente está dispuesto esperar, no importa lo barato que cueste el producto y el precio máximo que pagaría aunque la entrega sea inmediata, permite al entrenado decidir la mejor estrategia comercial teniendo en cuenta su impacto no sólo en el mercado que gana sino también en sus costos y performance logística.

Este modelo permite la retroalimentación de las cadenas en competencia, que tienen por objetivo, al igual que en otros juegos, atender a los pedidos de los clientes con 
el mejor Nivel de Servicio y el Costo Logístico Total más bajo, pero que les permita ganar una porción de mercado adecuada. El Modelo de Demanda trae un cambio de foco en el objetivo del juego, siendo más representativo de la realidad.

\section{Referencias:}

1. Lambert, D.M., Cooper, M.C., and Pagh, J.D.: Supply Chain Management: Implementation Issues and Research Opportunities. Int. J. Logistics Manage. 9, 1-20 (1998)

2. Sterman, J. D.: Teaching Takes Off: Flight Simulators for Management Education. OR/MS Today, 40-44 (1992)

3. Leger, P.M.: Using a simulation game approach to teach ERP concepts. HEC Montre'al, Groupe de recherche en syste'mesd'information, Montre'al. (2006)

4. Petranek, C.: Amaturation in experiential learning: Principles of simulation and gaming. SimulatGaming.Int J 25, 513 522. (1994)

5. Crown, S.W.: Improving visualization skills of engineering graphics students using simple JavaScript web based games. J EngEduc 90, 347_355.(2001)

6. Torres, M. and Macedo, J.: Learning sustainable development with a new simulation game. Simulat Gaming 31, 119_126.(2000)

7. Deshpande, A. and Huang, S.: Simulation games in engineering education: A state-of-theart review. Comp. Appl. Eng. Educ. (en prensa). (2009)

8. Riis, J.O., Johansen, J. and Mikkelsen, H.: Simulation Games and Learning in Production Management. Chapman \& Hall, London, UK., 1-12. (1995)

9. Ravid, G., Rafaeli, S.: Multi Player, Internet and Java-Based simulation Games: Learning and research in implementing a computerized version of the "Beer-distribution supply chain game". (2000)

10. Zhang, D.: A network economic model for supply chain versus supply chain competition. The International Journal of Management Science. 34, 283-295. (2006)

11. Gold, S. and Pray, T.: Modeling Demand in Computerized business simulations. in: Guide to business gaming and experiential learning. J. Gentry, ABSEL, 117-138. (1990)

12. Sterman, J.: Modeling managerial behavior: misperceptions of feedback in a dynamic decision making experiment. Management Science, 35, 321-339. (1989)

13. Kimbrough, S.O., Wu, D.J., Zhong, F.: Computers play the beer game: can artificial agents manage supply chains?. Decision Support Systems, 33, 323- 333. (2002)

14. Thorsteinsson, U.: The LOGI-Game: a dynamic modular logistic game. in: J. O. Riis, Simulation Games and Learning in Production Management. Chapman \& Hall, London, UK, 176-183. (1995).

15. Technical University of Denmark, http://www.moltho.dk

16. Chang Y. C, Chen W. C.: A flexible web-based simulation game for production and logistics management courses. SIMPRA 17, 1241-1253. (2009)

17. Feng and Ma: Learning Supply Chain Management with Fun: An Online Simulation Game Approach. California Journal of Op. Management Vol. 6, No. 1, pp 41-48. (2008)

18. Arunachalam R. and Sadeh N.M.: The Supply Chain Trading Agent Competition. Electronic Commerce Research and Applications 4, 66-84. (2005)

19. Tsay, A., Agrawal, N.: Channel dynamics under price and service competition. Manufacturing and Service Operations Management. Vol. 2, No 4, 372-391. (2000) 